\title{
Apoyando el despliegue de procesos en el contexto de las pequeñas organizaciones software
}

\author{
Supporting Process Deployment in the Context of Small Software Organizations
}

Apoiar a implantação de processos no contexto de pequenas organizações de software

\author{
Francisco Zambrano ${ }^{1}$ \\ Nelson Patiño \\ Francisco-José Pino-Correa ${ }^{3}$ (D)
}

Recibido: agosto de 2021

Aceptado: octubre de 2021

Para citar este artículo: Zambrano, F., Patiño, N., y Pino-Correa, F. J. (2022). Apoyando el despliegue de procesos en el contexto de las pequeñas organizaciones software. Revista Científica, 43(1), 50-63. https://doi. org/10.14483/23448350.18351

\section{Resumen}

El despliegue de procesos software es una labor de las organizaciones para asegurar que los procesos se usen y sean seguidos en el desarrollo de sus proyectos. Sin embargo, la mayoría de las propuestas que existen para el despliegue de procesos son abstractas, difíciles de aplicar, y no consideran las limitaciones de tiempo y recursos propias de las pequeñas empresas. En este artículo se presenta un método para apoyar el despliegue de procesos en el contexto de las pequeñas organizaciones desarrolladoras de software, basado en elementos de internalización de conocimiento explícito y gestión de resistencia al cambio. El método propuesto ha sido evaluado mediante expertos, considerando aspectos de adaptabilidad, comprensibilidad e idoneidad del método para su uso en pequeñas organizaciones de software. A partir de esta evaluación se puede concluir que el método es ligero, su descripción es detallada y puede ser aplicado en organizaciones de este tipo.

Palabras clave: despliegue de procesos; gestión de conocimiento; internalización; proceso software.

\begin{abstract}
Software process deployment is a work carried out by organizations to ensure that a process is used and followed in the development of their projects. However, most existing proposals for process deployment are abstract, hard to apply, and do not consider the time and resource limitations of small enterprises. In this article, a method to support process deployment in the context of small software organizations is introduced, based on elements of explicit knowledge internalization and change management. The proposed method has been assessed by experts, considering aspects of adaptability, understandability, and
\end{abstract}

1. Universidad del Cauca. Popayán, Colombia. avogfjzj@unicauca.edu.co.

2. Universidad del Cauca. Popayán, Colombia. npatino@unicauca.edu.co.

3. Universidad del Cauca. Popayán, Colombia. fjpino@unicauca.edu.co. 
suitability of the method for use in small software organizations. From this assessment, we can conclude that the method is a lightweight proposal, its description is detailed, and it can be applied in such organizations.

Keywords: internalization; knowledge management; process deployment; software process.

\section{Resumo}

A implantação de processos de software é um trabaIho das organizações para garantir que os processos sejam utilizados e acompanhados no desenvolvimento de seus projetos. No entanto, a maioria das propostas existentes para implantação de processos são abstratas, difíceis de aplicar e não consideram as limitações de tempo e recursos de pequenas empresas. Neste artigo, é apresentado um método para apoiar a implantação de processos no contexto de pequenas organizações de software, baseado em elementos de internalização de conhecimento explícito e gerenciamento de mudanças. O método proposto foi avaliado por especialistas, considerando aspectos de adaptabilidade, compreensibilidade e adequação do método para uso em pequenas organizações de software. A partir dessa avaliação, podemos concluir que o método é uma proposta leve, sua descrição é detalhada e pode ser aplicado em tais organizações.

Palavras-chaves: processo de software, implementação de processos, gestão do conhecimento, internalização

\section{Introducción}

Cuando se trata de mejora de procesos software, el despliegue de procesos es un tema fundamental, el cual está orientado a llevar a la práctica los procesos que define una organización para el desarrollo de sus proyectos. Formalmente, el despliegue de procesos se define como "el proceso que permite la implementación, gestión, adopción e institucionalización de los procesos definidos en la ingeniería de procesos" (Bayona-Oré et al., 2008), y se centra en la forma de asegurar que las personas sigan el proceso y lo usen en sus tareas diarias (Forrester, 2006). Aunque parece un trabajo trivial, el despliegue de procesos no puede ser considerado como tal; las compañías requieren de métodos formales que les permitan realizar adecuadamente su trabajo (Cornu, Chapurlat, Quiot e Irigoin, 2012). Actualmente existen pocos métodos que describen cómo desplegar procesos, los cuales son complejos y desconocidos para la mayoría de las compañías (Cornu et al., 2012).

Si un proceso se despliega adecuadamente en un proyecto, se espera que con el tiempo continúe desplegándose exitosamente en todos los demás proyectos de la organización, es decir, se espera que el proceso se institucionalice. La institucionalización implica que el proceso se arraiga en la forma de realizar el trabajo, de tal manera que exista compromiso y consistencia en su ejecución (SEI, 2010). En este sentido, la institucionalización de un proceso organizacional se puede definir como el trabajo de convertir en hábito las buenas prácticas, con el fin de asegurar que su cumplimiento sea continuo y permanente, aún en los tiempos de crisis (Garzás, Pino, Piattini y Fernández, 2013). Según un estudio realizado en 16 firmas españolas de software (de las cuales, el $75 \%$ consiste en empresas con no más de 50 empleados), la falta de institucionalización es un problema común entre las pequeñas empresas de software (Garzás et al., 2013). Esta falta de institucionalización señala la dificultad que tienen las pequeñas organizaciones (VSE, por sus siglas en inglés, cuya característica es que tienen menos de 25 empleados) para ejecutar sus procesos de forma adecuada y consistente en sus proyectos, lo cual les impide mantener los niveles de capacidad de sus procesos, afectando negativamente su calidad y la de sus productos.

Como una solución al problema de la falta de institucionalización, existen modelos de referencia en los cuales se recomienda seguir sus prácticas para ayudar a las organizaciones a institucionali-

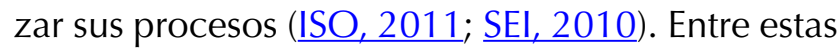
prácticas se encuentran aquellas relacionadas con el despliegue de procesos, que consiste en poner en práctica las definiciones de los procesos documentados en la organización (Forrester, 2006). 
Algunos autores plantean que para desplegar procesos se deben tener en cuenta diferentes aspectos técnicos y sociales de la organización que parecen aislados entre sí (Bayona, Calvo-Manzano, Cuevas y San Feliu, 2010; Montoni et al., 2006). Si bien es cierto que es importante considerar estos aspectos en la construcción de una propuesta para la ejecución consistente de los procesos, el problema puede persistir si dicha propuesta no es fácil de emplear, o puede no ser adecuada para una pequeña organización al requerir de una gran cantidad de tiempo y recursos. Entonces la pregunta de investigación a abordar en este trabajo es: ¿cómo apoyar a las pequeñas organizaciones a ejecutar sus procesos definidos en los proyectos que estas realicen? En este sentido, el presente artículo introduce un método para el despliegue de procesos dirigido a pequeñas organizaciones desarrolladoras de software. El objetivo de este método, denominado MEDEPRO, es apoyar la ejecución de los procesos que define una pequeña organización en sus proyectos, mediante una propuesta ligera, con pocos elementos y que consuma pocos recursos, para ajustarse a sus limitaciones. Para apoyar el despliegue, este método se basa en elementos de internalización de conocimiento explícito, con el fin de ayudar a las personas a adquirir el conocimiento tácito que necesitan para desarrollar habilidades y crear nuevo conocimiento en la organización. El método propuesto ha sido evaluado mediante expertos, considerando aspectos de adaptabilidad, comprensibilidad e idoneidad del método para su uso en pequeñas organizaciones de software. A partir de esta evaluación se puede concluir que el método es ligero, su descripción es detallada y puede ser aplicado en organizaciones de este tipo.

El artículo se estructura de la siguiente manera: en la sección de metodología se presentan los trabajos relacionados y la estrategia de investigación empleada; en la sección de resultados se describe el método propuesto y su evaluación mediante la estrategia de investigación Focus Group, finalmente se presentan las conclusiones.

\section{Metodología}

\section{Estrategia de investigación}

La naturaleza de este trabajo está orientada hacia la construcción de un método que permita la adopción de procesos y su ejecución en proyectos, enfocado hacia el contexto de las pequeñas organizaciones desarrolladoras de software. Por esta razón, luego de estudiar algunos métodos investigativos propuestos por Marcos (2002), McKay y Marshall (2001) y Serrano (2009), se definió una estrategia de investigación ad-hoc que se ajusta adecuadamente a los objetivos de este trabajo. A continuación, se presentan las fases que fueron definidas para la construcción del método propuesto:

\section{- Fase 1: Análisis documental}

En esta fase inicialmente se recolectó y estudió información a partir de libros, monografías, tesis, artículos, informes, revistas y ensayos referentes a los temas de institucionalización de procesos, despliegue de procesos, pequeñas organizaciones desarrolladoras de software, internalización del conocimiento, creación de métodos, definición de modelos y evaluación de propuestas, entre otros. Posteriormente, se realizó un análisis de cada una de las propuestas relacionadas con el despliegue de procesos y la internalización del conocimiento explícito, con el fin de obtener elementos de despliegue apropiados para pequeñas organizaciones desarrolladoras de software. Por último, se compararon las propuestas para extraer los elementos comunes entre ellas.

\section{- Fase 2: Definición del método}

En esta fase se sintetizaron las actividades, técnicas y herramientas identificadas anteriormente y se justificó su selección para incluirlas en el método de despliegue. Con los elementos seleccionados y justificados, se construye la estructura del método y el flujo de actividades. Por último, el 
método y sus elementos se describieron detalladamente, relacionando estos últimos entre sí.

\section{- Fase 3: Modelado del método}

En esta fase se realizó una representación del método en SPEM 2.0 mediante la herramienta Eclipse Process Framework Composer. Inicialmente, se crearon y se categorizaron los elementos que son parte del contenido del método. Acto seguido, se formó el proceso de despliegue mediante un conjunto de actividades ordenadas y compuestas por los elementos creados anteriormente. Finalmente, se estableció una configuración del método, la cual se publicó para desarrollar una guía electrónica de procesos con el fin de apoyar al método de despliegue.

\section{- Fase 4: Evaluación y análisis de resultados}

La evaluación del método de despliegue se realizó empleando Focus Group, una estrategia útil para la evaluación de propuestas teóricas a partir del juicio de expertos (Mendoza, González y Pino, 2013). La estrategia en la evaluación de la propuesta se realizó de la siguiente manera: primero se conformó un grupo de discusión con expertos en el tema de despliegue de procesos en pequeñas organizaciones, quienes debatieron sobre las debilidades y fortalezas de la propuesta. Todas las observaciones de los expertos obtenidas en el debate se analizaron para identificar los cambios que es necesario realizar en el método propuesto. Por último, se aplicaron dichos cambios para elaborar una nueva versión del método.

\section{Trabajos relacionados}

En esta investigación se estudiaron dos modelos de referencia internacional que contienen elementos para el despliegue de procesos descritos en alto nivel de abstracción. El primer referente estudiado es el modelo ejemplar de evaluación de procesos del ciclo de vida del software, conocido como ISO/IEC FDIS 15504-5:2012, el cual contiene el atributo
PA.3.2, definido como una medida del grado en el que un proceso estándar se despliega efectivamente como un proceso definido (ISO, 2012). El segundo modelo de referencia internacional estudiado es el CMMI para el desarrollo versión 3, el cual contiene, dentro del área de procesos conocida como Foco de Proceso Organizacional (OPF), un conjunto de prácticas relacionadas con el despliegue de procesos, asociadas al objetivo específico SG3, "Desplegar activos de procesos organizacionales e incorporar experiencias" (SEI, 2010).

Además de estos dos modelos de referencia, se encontraron estudios que abordan el despliegue de procesos software, los cuales se muestran a continuación:

- Kaltio y Kinnula (2000) presentan un modelo para el despliegue de procesos enfocado en la gestión de activos de procesos organizacionales. Según sus autores, el despliegue de procesos implica un conjunto de actividades tales como selección de activos de procesos, adaptación de los activos a las necesidades específicas de los proyectos, capacitación del personal, adquisición e instalación de recursos, promulgado y revisión del promulgado.

- Bayona et al. (2010) presentan una taxonomía de factores críticos de éxito para el despliegue de procesos. En esta taxonomía, la institucionalización de un proceso es el resultado de su despliegue exitoso y está condicionado por el cumplimiento de los factores críticos propuestos. Según sus autores, aquellos factores de éxito relacionados con los aspectos sociales de la organización son los más importantes, destacando el compromiso de alta dirección en las actividades de despliegue de procesos, la formación del personal y la comunicación entre los distintos niveles jerárquicos de la organización.

- Cornu et al. (2012) presentan un modelo de madurez para evaluar la preparación de las organizaciones en el momento de desplegar sus procesos. Este modelo se basa en la suposición de que la interoperabilidad (definida como la 
habilidad de trabajar juntos eficientemente mediante una comunicación efectiva) es un factor clave en el despliegue de procesos. El propósito del modelo es ayudar a encontrar las debilidades de las grandes organizaciones que amenazan el éxito del despliegue de sus procesos. Estas debilidades se dividen en tres clases de problemas (denominados barreras) relacionados con la interoperabilidad entre los recursos de las organizaciones: (i) barrera conceptual, agrupa aquellos problemas concernientes a la expresión y la representación del conocimiento; (ii) barrera tecnológica, consiste en problemas relacionados con el uso de herramientas que impiden la colaboración entre las personas, y (iii) barrera organizacional, consiste en problemas que surgen a la hora de establecer responsabilidades y ejercer autoridades.

- Cornu, Chapurlat, Irigoin y Quiot (2012) proponen un método para el despliegue de procesos enfocando en el uso de elementos tanto de ingeniería de sistemas como de modelado de empresas. Al igual que en el modelo de madurez, el método se enfoca en la solución de problemas relacionados con la interoperabilidad, promoviendo la creación de un lenguaje de despliegue común (en forma de metamodelo) que unifique los conceptos relacionados con el diseño de productos y actividades.

- Montoni et al. (2006) presentan una infraestructura para la definición, el despliegue y el promulgado de procesos estándares organizacionales y procesos software, basada en la integración de modelos de madurez de capacidades (CMMI) y en el modelo de referencia para la mejora de procesos software de Brasil (MR-MPS). Los desarrolladores de esta infraestructura aseguran que la institucionalización de los procesos organizacionales se obtiene por medio de la sistematización de las tareas que consideran más importantes en la ingeniería de procesos, como la definición de los procesos, la gestión de proyectos y la recolección de medidas en los proyectos.
La mayoría de las propuestas de despliegue están dirigidas a las organizaciones software sin considerar si es aplicable a su tamaño. El método propuesto en este artículo está dirigido a pequeñas organizaciones, descartando aquellos elementos que, debido a su complejidad y alto nivel de abstracción, dificultan su aplicación en este tipo de organizaciones. También se observa que la mayoría de las propuestas no proveen plantillas que apoyen los procedimientos para el despliegue de los procesos. Las plantillas son uno de los elementos preferidos por los ingenieros de software porque son fáciles de usar y contribuyen a un mejor aprendizaje de las tareas (Sanchez-Segura, Medina-Dominguez, Amescua y Mora-Soto, 2010). Por tal motivo, el método propuesto provee plantillas que guíen los procedimientos para desplegar fácil y efectivamente los procesos organizacionales.

Por último, en los trabajos relacionados se considera vital la gestión de conocimiento en el despliegue de procesos. No obstante, estas propuestas se limitan a obtener, documentar y almacenar información en una base de datos organizacional para usos posteriores. El método propuesto en este artículo también se basa en la gestión de conocimiento, pero, a diferencia de las otras propuestas, en la internalización de conocimiento explícito, para que las personas se apropien de la información contenida en la descripción de los procesos definidos de la organización.

\section{Internalización del conocimiento}

La internalización es el proceso de transformar el conocimiento explícito contenido, por ejemplo, en los procedimientos de una empresa, en conocimiento tácito propio de la persona a través de la acción, la práctica y la reflexión (Nonaka y Toyama, 2003). Algunos autores sugieren, como técnicas de internalización, realizar entrenamientos durante el trabajo, tales como formación (coaching) y tutoría (mentoring) (Massey y Montoya-Weiss, 1997; Nonaka y Toyama, 2003; Wigg, 2004; Young, 2010), mientras que otros recomiendan el uso de wikis y 
otras herramientas que permitan realizar lecturas activas y a su vez compartir nueva información basada en la experiencia (Chikh, 2011; Sousa, Aparicio y Costa, 2010). El método propuesto en este artículo adapta elementos de los trabajos de internalización propuestos por Uchihira (2006), Uchihira et al. (2012) y Read (2008), Ilevándolos al contexto de la gestión de procesos para elaborar una estrategia que pueda ayudar a los trabajadores a internalizar el conocimiento explícito de los procesos software. Como soporte a esta estrategia, el método incluye dos técnicas de entrenamiento en el trabajo propuestas respectivamente por Walter (2001) y Holliday (2001), con el fin de realizar un seguimiento de los empleados durante las instrucciones sobre el proceso organizacional.

\section{Resultados}

\section{Método de despliegue MEDEPRO}

Un método es un conjunto de actividades coherentes con el fin de llevar a cabo un propósito específico (Oktaba, Morales y Dávila, 2012). El objetivo del método propuesto en este artículo es ayudar a las pequeñas organizaciones de software a desplegar sus procesos en proyectos particulares. A continuación, se presenta un resumen de la versión del método elaborada después de la evaluación.

\section{Propósito:}

El método MEDEPRO apoya el despliegue de procesos en los proyectos de pequeñas organizaciones desarrolladoras de software, procurando contribuir al esfuerzo de dichas organizaciones por institucionalizar sus procesos.

Esquema general:

Antes de usar el método, primero se identifica un nuevo proyecto de la organización a desarrollar, para el cual se selecciona un proceso organizacional cuyos resultados sean similares a los esperados por el tipo de proyecto identificado. Posteriormente, los cinco roles del método se asignan entre los miembros de la organización, teniendo en cuenta que una persona puede desempeñar muchos roles, o un rol puede ser desempeñado por muchas personas. La secuencia de las actividades del método de despliegue propuesto en ilustra en la Figura 1.

Roles:

Los roles que se asignan antes de emplear el método de despliegue se presentan a continuación:

- Líder de despliegue, responsable de la planeación, la asignación y el desarrollo de las actividades necesarias para el despliegue. Debe ser un miembro de la organización.

- Revisor, responsable de verificar la adecuada realización de las actividades del proceso definido en el proyecto.

- Facilitador de entrenamiento, responsable de ayudar a un grupo de personas para definir los objetivos de entrenamiento y la forma de alcanzarlos, de facilitar el acceso al conocimiento, de manera clara y ordenada, debe conocer los procesos de la organización.

- Entrenador, persona que instruye, forma y guía al aprendiz para que mejore en el desempeño de sus funciones, debe tener un conocimiento profundo del trabajo desarrollado por el aprendiz y conocer los objetivos de la organización.

- Ejecutor, responsable de llevar a cabo las tareas del proceso definido para su ejecución en un proyecto, durante la actividad de entrenamiento se desempeña como aprendiz y debe ser un miembro de la organización.

Productos de trabajo:

A continuación, se describen los productos de trabajo empleados en esta propuesta:

- Activos de proceso, son todos aquellos documentos relacionados con un proceso de la 


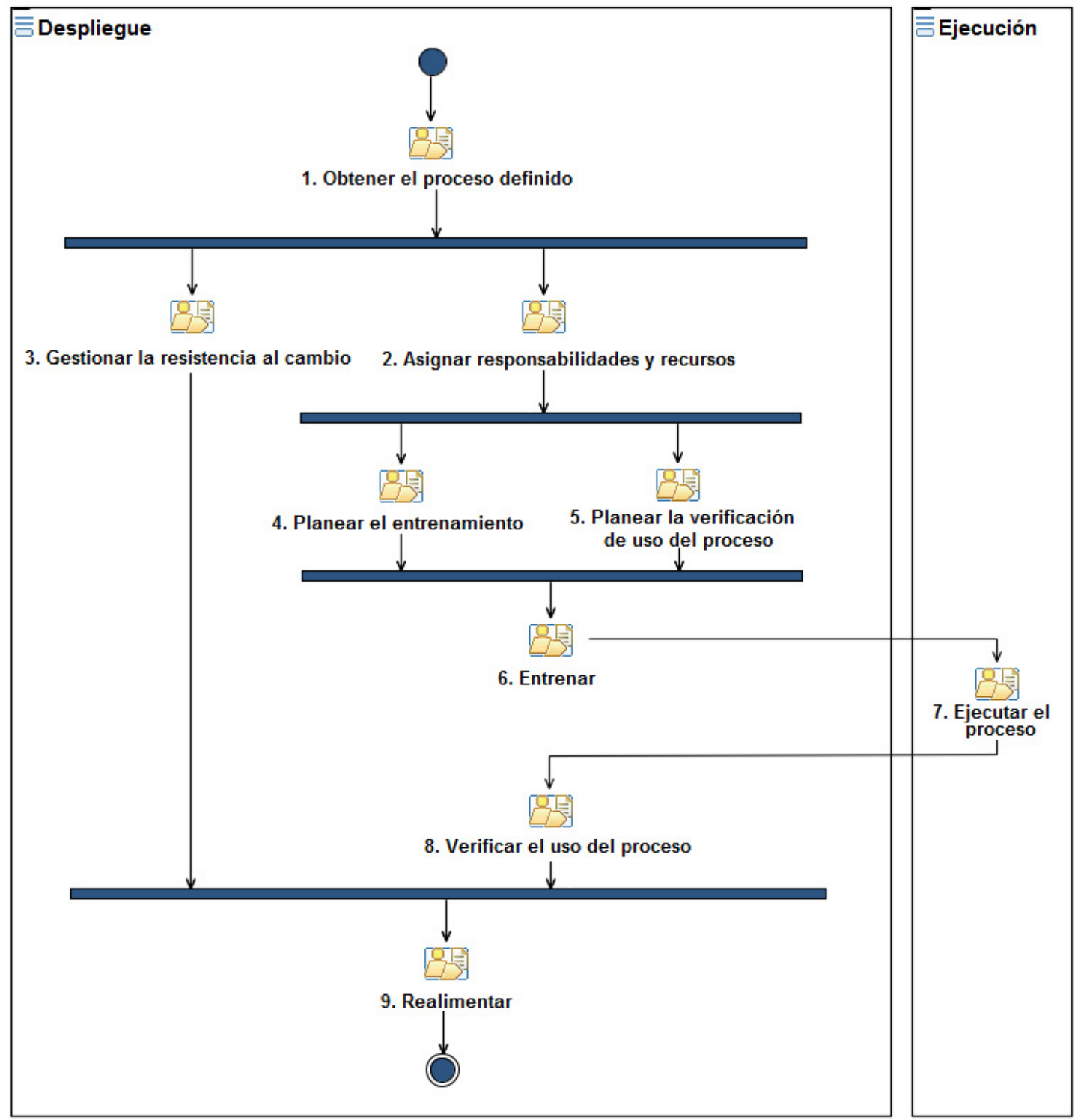

Figura 1. Diagrama de actividades del método MEDEPRO

Fuente: elaboración propia

organización que pueden ser empleados en un proyecto específico. Los activos de proceso se componen de: (i) proceso de la organización, (ii) proceso definido del proyecto, (iii) líneas guía de adaptación, (iv) cambios en el proceso de la organización, (v) revisión del proceso definido, y (vi) módulos de entrenamiento.

- Plan de despliegue, describe propósito, alcance y objetivos de la gestión de resistencia al cambio del personal, el entrenamiento y la verificación del uso del proceso en el proyecto actual. El plan de despliegue se compone de: (i) plan de gestión de resistencia al cambio, (ii) plan de entrenamiento de proceso, (iii) documento de análisis de entrenamiento, y (iv) plan de verificación de uso del proceso.

- Cronograma, registra los tiempos de ejecución de las tareas del proceso y los horarios para la realización de las sesiones de entrenamiento y las de verificación de uso del proceso. El 
cronograma se compone de: (i) comunicado de asignación de tareas y recursos, (ii) cronograma de sesiones de entrenamiento, (iii) documento de asignaciones de entrenamiento, y (iv) cronograma de reuniones de verificación.

- Realimentación, registra los resultados del despliegue de un proceso en un proyecto para ayudar a tomar decisiones en la planeación de futuros despliegues. La realimentación se compone de: (i) perfiles de ejecutores, (ii) evaluación de módulo de entrenamiento, (iii) verificación del uso de tareas, y (iv) documento de lecciones aprendidas.

A excepción del proceso definido del proyecto, el proceso de la organización y las líneas guía de adaptación del producto de trabajo activos de proceso, el método provee plantillas para todos los subproductos de trabajo con el fin de facilitar la gestión del despliegue. Los tres subproductos de trabajo mencionados anteriormente no tienen plantillas porque su formato depende de la organización.

Descripción general de las actividades:

- Obtener el proceso definido: se adapta un proceso organizacional para formar el proceso definido que satisfaga la necesidad particular de un proyecto. Entre sus actividades se encuentran: (i) internalizar el proceso de la organización, (ii) adaptar el proceso de la organización, y (iii) revisar la descripción del proceso definido del proyecto.

- Asignar responsabilidades y recursos: se asignan las tareas y los roles del proceso definido del proyecto al personal junto con los recursos requeridos para realizarlas. En esta actividad se realizan las tareas de: (i) comunicar las responsabilidades a las personas encargadas de ejecutar el proceso, y (ii) proveer los recursos que necesitan para el desarrollo de sus tareas.

- Gestionar la resistencia al cambio: se comprende por qué es necesario el cambio que involucra el despliegue, y se identifica a las personas afectadas para reducir la resistencia al cambio. Esta actividad consta de las siguientes tareas: (i) establecer la visión de resistencia al cambio, (ii) analizar el contexto del cambio, e (iii) introducir el cambio.

- Planear el entrenamiento: se identifican, analizan y detallan los elementos necesarios para llevar a cabo el entrenamiento del personal. Esta actividad contiene las siguientes tareas: (i) determinar la visión de entrenamiento, (ii) analizar los módulos de entrenamiento existentes, y (iii) diseñar los módulos de entrenamiento requeridos.

- Planear la verificación del uso del proceso: se establecen criterios y soporte para evaluar el seguimiento del proceso por parte de los empleados. En esta actividad solo se realiza el plan de verificación.

- Entrenar: se ayuda al personal a desarrollar habilidades y a adquirir los conocimientos requeridos en su trabajo. Esta actividad consta de las siguientes tareas: (i) asignar entrenadores y módulos de entrenamiento, (ii) programar las sesiones de entrenamiento, (iii) conducir las sesiones de entrenamiento, y (iv) verificar el entendimiento.

- Ejecutar el proceso: se realizan las tareas que han sido asignadas a los ejecutores en el desarrollo del proyecto. La forma de realizar las tareas puntuales del proceso se encuentra fuera del alcance de este método.

- Verificar el uso del proceso: se comprueba que el personal sigue el proceso y los productos de trabajo que realiza son adecuados. En esta actividad se realizan las siguientes tareas: (i) programar las reuniones de verificación, y (ii) realizar las reuniones de revisión.

- Realimentar: se documentan las lecciones aprendidas en el despliegue del proceso para emplearlo en futuros proyectos.

- Descripción detallada de una tarea: debido a restricciones de espacio, a continuación, se presenta solo una tarea del método. Para ver 
la propuesta completa, diríjase a Patiño y Zambrano (2014a) y a la guía electrónica de proceso disponible (Patiño y Zambrano, 2014b). A continuación, en la Tabla I, se presenta la descripción de esta tarea:

\section{Evaluación de método MEDEPRO}

Para evaluar el método propuesto, se sometió la
propuesta al juicio de expertos en los temas de despliegue de procesos y gestión de conocimiento, empleando el método de investigación cualitativa Focus Group. El objetivo principal de la evaluación consistió en obtener información acerca del grado de aceptación o rechazo por parte de los expertos que participaron en una sesión de debate, enfocándose en los aspectos de idoneidad, comprensibilidad y adaptabilidad de la propuesta en pequeñas organizaciones de software. La evaluación se llevó a cabo teniendo en cuenta la estructura teórica

Tabla I. Descripción detallada de la tarea Internalizar el proceso

Nombre: Tarea 1.1. Internalizar el proceso de la organización

Descripción: Esta tarea consiste en comprender mejor el proceso organizacional para luego especificar los cambios que se le realizarán, según las necesidades del proyecto. Para ello, se reúnen a todas las personas involucradas en el despliegue (especialmente ejecutores), se les comparte información relacionada con el uso previo del proceso organizacional y se discuten las necesidades. Por último, se documentan los cambios acordados.

Producto de trabajo de entrada: Activos de proceso (Proceso de la organización).

Producto de trabajo de salida: Activos de proceso (Cambios en el proceso de la organización).

Pasos:

Paso 1. Recolectar todos los documentos relacionados con el proceso de la organización: se recopila toda la documentación que describa el diseño y el comportamiento operacional del proceso organizacional. El proceso debe existir y estar explícitamente documentado, bien sea en forma impresa o en forma digital.

Paso 2. Obtener información y experiencia a partir de una base organizacional: se recolecta toda aquella información relacionada con la forma de realizar un tipo de proyecto en particular. Esta información puede encontrarse en los documentos de lecciones aprendidas en despliegues de procesos anteriores, experiencias del personal y juicios de expertos en general.

Paso 3. Convocar al personal involucrado: se reúne a todas las personas que formen parte en el despliegue del proceso, en especial aquellas involucradas directamente en su ejecución. Lo primero que se debe realizar en la reunión es presentar el proceso organizacional junto con la experiencia recolectada sobre despliegues de procesos en proyectos similares a los nuevos proyectos a desarrollar.

Paso 4. Elaborar supuestos iniciales sobre los cambios: una vez se han presentado el proceso organizacional y la información sobre despliegues pasados, todo el personal encargado de ejecutar el proceso debe proponer los cambios que cree convenientes para realizar en el proceso, dependiendo del tipo de proyecto a desarrollar.

Paso 5. Compartir experiencias: se abre un espacio para que las personas compartan su experiencia sobre la ejecución o adaptación del proceso en proyectos similares y sus supuestos para el proyecto actual; la narración (storytelling) y la realización de preguntas abiertas pueden emplease para este fin.

Paso 6. Discutir sobre el conocimiento adquirido: se estudian las discusiones en grupo y se debate sobre las ventajas y desventajas de las mismas. La idea de la discusión es comprender grupalmente los casos de despliegue anteriores Paso 7. Elaborar nuevos supuestos: después de discutir y revisar el proceso, se realizan mejoras individualmente a los cambios supuestos y se discuten de nuevo en grupo.

Paso 8. Documentar los cambios acordados en el proceso de la organización: después de discutir los nuevos supuestos y llegar a un consenso, todos los puntos acordados se documentan en el documento de cambios en el proceso de la organización. Si todos los involucrados acuerdan no realizar ningún cambio en el proceso organizacional, bien sea porque el proceso cumple todas las expectativas del proyecto o ya ha sido desplegado antes, debe hacerse explícito en el documento.

Fuente: elaboración propia 
descrita en Kontio, Bragge y Lehtola (2008) y el proceso propuesto en Mendoza et al., 2013.

Durante la fase de planeación, se definieron los objetivos de la evaluación y se asignaron los roles de moderador (encargado de dirigir la sesión de debate), relator (encargado de capturar la información de los participantes) y supervisor (quien observa y es el responsable de la evaluación). Posteriormente, se definieron los documentos necesarios para registrar la información obtenida durante la evaluación y las herramientas empleadas en la recolección de dicha información. También se definieron los aspectos que se deben evaluar en la propuesta, los cuales fueron obtenidos y adaptados a partir de la caracterización de los atributos de calidad del software propuestos en el referente internacional ISO/IEC FDIS 9126-1 (ISO, 2001). Los aspectos del método propuesto tenidos en cuenta para la evaluación fueron los siguientes:

- Idoneidad: es la capacidad del método para proveer un conjunto apropiado de funciones para tareas específicas y objetivos del usuario, los cuales están orientados a ser adecuados para pequeñas organizaciones desarrolladoras de software. Los objetivos de este aspecto son: (i) ser una propuesta ligera en términos de pocas actividades, pocos roles y pocos productos de trabajo, (ii) fortalecer la comunicación, la toma de decisiones y la resolución de problemas de manera informal, (iii) adaptarse a las limitaciones de recursos económicos de la organización, y (iv) apoyar el despliegue de procesos en los proyectos de la organización.

- Comprensibilidad: es la capacidad del método para permitirle al usuario entender si el método es adecuado, y ayudarle a comprender cómo puede ser usado bajo ciertas condiciones.

- Adaptabilidad: es la capacidad del método para ser adaptado en diferentes ambientes específicos sin aplicar acciones o medios distintos de aquellos provistos para el propósito del método propuesto.
Con los aspectos de evaluación definidos, se procedió a formular las preguntas de la encuesta que fue diligenciada por los participantes al terminar la sesión de debate. A cada pregunta se le asignó un identificador único y se asoció con un aspecto de evaluación. Las tres primeras preguntas del cuestionario, identificadas respectivamente como 1.1, 1.2 y 1.3, fueron formuladas para evaluar cada una de las actividades del método, mientras que las demás preguntas se formularon para evaluarlo de forma general. Las preguntas de la encuesta y los aspectos que evalúan se observan en la Tabla II.

Posteriormente, se seleccionaron los participantes que formaron parte de la sesión de debate. Para la selección, se planteó una estrategia que permitiera estudiar el perfil de los posibles participantes y se determinaron criterios para decidir si las personas podían participar en el debate, conforme a su experiencia en los temas de despliegue de procesos y gestión de conocimiento. De dieciséis posibles participantes, doce cumplieron los criterios establecidos, pero solo cuatro confirmaron su asistencia, y con ellos se realizó la evaluación de la propuesta. Los cuatro participantes son expertos en la industria del software y están relacionados con el tema de la gestión de conocimiento en empresas.

La sesión de debate inició con la presentación del protocolo y del método de despliegue, enfocándose en los objetivos de la propuesta y la descripción general de los elementos en que se compone. En seguida, a cada participante se le otorgó la palabra, para que compartiera sus observaciones al resto del grupo a partir de sus experiencias laborales. Durante la sesión se realizaron grabaciones y anotaciones sobre los puntos que trataron los participantes y, una vez terminada, se les entregó el cuestionario para complementar sus observaciones. Por último, la información recolectada se analizó para corregir el método de despliegue y elaborar una nueva versión del mismo, la cual fue presentada en la sección anterior. 
Tabla II. Preguntas del cuestionario para la evaluación del método propuesto

\begin{tabular}{|c|c|c|}
\hline Id & Pregunta & Aspecto a evaluar \\
\hline 1.1 & ¿El número de tareas que contiene la actividad es adecuado para una pequeña organización? & Idoneidad, objetivos 1 y 3 \\
\hline 1.2 & ¿La actividad es útil en el desarrollo de proyectos de una organización? & Idoneidad, objetivo 4 \\
\hline 1.3 & ¿La descripción de la actividad es comprensible? & Comprensibilidad \\
\hline 2.1 & $\begin{array}{l}\text { ¿Considera que la cantidad de actividades del método propuesto es adecuada para pequeñas } \\
\text { organizaciones? }\end{array}$ & Idoneidad, objetivos 1 y 3 \\
\hline 2.2 & ¿Considera que la cantidad de roles del método propuesto es adecuada para pequeñas organizaciones? & Idoneidad, objetivos 1 y 3 \\
\hline 2.3 & $\begin{array}{l}\text { ¿Considera que la cantidad de productos de trabajo del método propuesto es adecuada para pequeñas } \\
\text { organizaciones? }\end{array}$ & Idoneidad, objetivos 1 y 3 \\
\hline 2.4 & ¿Considera que las descripciones de los roles son comprensibles? & Comprensibilidad \\
\hline 2.5 & ¿Considera que los diagramas empleados en las plantillas son comprensibles? & Comprensibilidad \\
\hline 2.6 & ¿Considera que las plantillas del método estructuran adecuadamente la información? & Comprensibilidad \\
\hline 2.7 & $\begin{array}{l}\text { ¿Considera que la aplicación de este método puede ayudar a los empleados a apropiarse fácilmente de } \\
\text { los procesos de una organización? }\end{array}$ & Idoneidad, objetivo 4 \\
\hline 2.8 & $\begin{array}{l}\text { ¿Consideraría usted que el método propuesto apoya y mejora la comunicación informal entre los } \\
\text { interesados del despliegue? }\end{array}$ & Idoneidad, objetivo 2 \\
\hline 2.9 & ¿Considera que este método es fácilmente adaptable? & Adaptabilidad \\
\hline 2.10 & ¿Qué ventajas y desventajas puede tener la aplicación de este método en una organización? & Adaptabilidad, Idoneidad \\
\hline
\end{tabular}

Fuente: elaboración propia

\section{Resultados de la evaluación}

A partir de la información recolectada del debate, se extrajeron veintitrés oportunidades de mejora para el método de despliegue, de las cuales catorce estaban relacionadas con las actividades, cuatro con los roles y productos de trabajo, y cinco con la propuesta en general. Entre estas oportunidades se encontraron mejoras en descripciones de los elementos existentes, aclaraciones sobre el uso, condiciones y ventajas del método, refinamiento de algunos pasos, y la inclusión de nuevos elementos. Durante la sesión, los participantes afirmaron que el método para el despliegue de procesos propuesto es un trabajo interesante porque busca ayudar a las pequeñas organizaciones a usar sus procesos de forma no empírica, y el nivel de detalle de las explicaciones es adecuado para ellas. Además, algunos participantes resaltaron que las actividades de realimentación y de verificación del uso del proceso son vitales en el despliegue, y reconocieron que los formatos empleados en las plantillas de los productos de trabajo son adecuados, fáciles de diligenciar y pueden ayudar a evitar una sobrecarga de trabajo.

De la encuesta diligenciada por los participantes, después de conducir la sesión de debate, se obtuvo lo siguiente:

- En la pregunta 1.1 todos los participantes estuvieron de acuerdo en que cinco actividades contienen pocas tareas, lo cual es adecuado para una pequeña organización, mientras que para dos actividades (entrenar y obtener el proceso definido), tres de cuatro participantes consideraron que sí contienen pocas tareas. En cambio, para la actividad de planear el entrenamiento, solo dos de cuatro participantes consideraron que el número de tareas es adecuado, razón por la cual esta fue una de 
las primeras actividades en modificarse para simplificar su trabajo. De la pregunta 1.2 se obtuvo que todas las actividades del método pueden ser útiles en el desarrollo de proyectos organizacionales. Solo una de cuatro personas consideró que la actividad de entrenar no es útil, mientras que todas estuvieron de acuerdo en que las demás actividades sí son útiles en el despliegue de procesos. De forma similar, en la pregunta 1.3 se obtuvo que las actividades son fáciles de entender y su nivel de detalle es adecuado. Solo una de cuatro personas sostuvo que la actividad de entrenar al personal no es comprensible. Aun así, se realizaron modificaciones en esta actividad para eliminar elementos que puedan dificultar su entendimiento.

- Las preguntas 2.1, 2.2, y 2.3 se agruparon para evaluar el aspecto de idoneidad del método propuesto conforme a los objetivos de idoneidad 1 y 3 . En las preguntas 2.1 y 2.2 todos los participantes afirman que la cantidad de actividades y roles del método de despliegue propuesto es adecuada para una pequeña organización. Sin embargo, en la pregunta 2.3 todos los participantes están de acuerdo en que el número de productos de trabajo no es conveniente para una organización de este tipo. Durante las discusiones, los participantes explicaron que en las pequeñas organizaciones no es común llenar una gran cantidad de documentos porque esto dificulta las tareas y retrasa los proyectos. Por tal motivo, en la nueva versión del método se eliminaron algunos productos de trabajo y, debido a que estos no son extensos, los definitivos se agruparon para facilitar su uso y comprensión. De forma similar, las preguntas 2.4, 2.5, y 2.6 se agruparon para evaluar la comprensibilidad de la propuesta. Todos los participantes afirman que las descripciones de los roles son comprensibles, los diagramas que se emplean para ilustrar las secuencias de actividades y tareas son fáciles de entender, y las plantillas del método utilizadas son adecuadas para estructurar adecuadamente su información.

- La pregunta 2.7 estuvo orientada a evaluar el objetivo 4 de idoneidad en la propuesta, con el cual se buscó verificar si el método propuesto es útil para el despliegue, en el sentido de que los empleados de la pequeña organización realmente puedan seguir el proceso definido durante el desarrollo de los proyectos. Todos los participantes estuvieron de acuerdo en que el método propuesto cumple con este aspecto. Con la pregunta 2.8 se evaluó el objetivo 2 del aspecto de idoneidad, el cual se refiere al fortalecimiento de la comunicación, la toma de decisiones y la resolución de problemas de manera informal. Todos los participantes afirmaron que el método puede ayudar a mejorar la comunicación entre las personas involucradas en el despliegue del proceso. En la pregunta 2.9 los cuatro participantes en la encuesta confirmaron que es posible adaptar el método en la organización para formar uno nuevo que se ajuste a sus necesidades particulares. La pregunta 2.10 fue la última pregunta del cuestionario y se formuló de forma abierta, en esta se les solicitó a los participantes, según su experiencia, mencionar qué ventajas o desventajas podría tener la aplicación de esta propuesta en una pequeña organización. Los resultados de esta pregunta son acordes a los obtenidos en el análisis de la información obtenida en la sesión de debate y las demás preguntas del cuestionario. Entre las ventajas del uso de este método se encuentran: (i) un despliegue ordenado y con mejores resultados, (ii) el mejoramiento del desempeño de los integrantes del proyecto, (iii) el cubrimiento adecuado de temas importantes en las organizaciones como gestión de resistencia al cambio, entrenamiento y realimentación, y (iv) el mantenimiento de la trazabilidad en los procesos. Dos de cuatro participantes señalaron como desventaja la resistencia que puede ocasionar su aplicación debido a la cantidad de productos de trabajo que implica. 


\section{Conclusiones}

Del análisis documental se concluyó que la mayoría de las propuestas para el despliegue de procesos pueden ser difíciles de aplicar en pequeñas organizaciones de software debido al alto nivel de abstracción en que se encuentran descritas y la gran cantidad de elementos que proveen sin tener en cuenta las limitaciones de este tipo de organizaciones. Del análisis también se concluye que no hay un consenso en el uso de ciertos términos en el área de ingeniería de procesos, tales como el mismo despliegue de procesos, y no existe una única forma de estructurar el desglose de los procesos como el de las propuestas para desplegarlos. En cuanto al tema de internalización de conocimiento explícito, también se observó que muchos autores sugieren emplear ciertas técnicas y herramientas de internalización, pero son pocos los que proponen una en concreto.

A partir de la evaluación se concluye que el método de despliegue de procesos MEDEPRO se considera útil, ligero, con pocos roles, pocas actividades, comprensible, adaptable, y el nivel de detalle de sus descripciones es adecuado para una pequeña organización no solo al indicar las tareas que deben realizarse (qué hacer), sino también la forma de realizarlas (cómo hacer). El método de Focus Group resultó ser eficaz para obtener de forma rápida las percepciones de un grupo de expertos sobre la propuesta de despliegue; sin embargo, para una evaluación más completa de la propuesta, se necesita llevar a cabo un estudio de caso en situaciones reales de las organizaciones o en proyectos piloto.

\section{Agradecimientos}

Francisco J. Pino agradece a la Universidad del Cauca, donde se desempeña como profesor titular del Departamento de Sistemas, además al Grupo de Investigación IDIS de la misma universidad.

\section{Referencias}

Bayona, S., Calvo-Manzano, J., Cuevas, G., San Feliu, T. (2010). Taxonomía de factores críticos para el despliegue de procesos software. REICIS, Revista Española de Innovación, Calidad e Ingeniería del Software, 6(3), 6-24

Bayona-Oré, S., Calvo-Manzano, J. A., Cuevas, G., San Feliu, T., Sánchez, A. (2008). Process deployment in a multi-site CMMI level 3 organization: A case study. In R. Lee \& H.-K. Kim (Eds.), Computer and Information Science Vol. 131 (pp. 147-156): Springer

Cornu, C., Chapurlat, V., Irigoin, F., Quiot, J.-M. (2012). Application of an enterprise modelling approach to deploy systems engineering processes in large organizations. Hyper Articles en Ligne, 1-25

Cornu, C., Chapurlat, V., Quiot, J.-M., Irigoin, F. (2012). A Maturity Model for the Deployment of Systems Engineering Processes. En IEEE International Systems Conference (SysCon 2012). https://doi. org/10.1109/SysCon.2012.6189535

Chikh, A. (2011). A knowledge management framework in software requirements engineering based on the SECl model. Journal of Software Engineering and Applications, 4(12), 718-728. https://doi. org/10.4236/jsea.2011.412084

Forrester, E. (Ed.). (2006). A Process Research Framework: The International Process Research Consortium. Pittsburgh, PA: Carnegie Mellon University, Software Engineering Institute

Garzás, J., Pino, F. J., Piattini, M., Fernández, C. M. (2013). A maturity model for the Spanish software industry based on ISO standards. Computer Standards \& Interfaces, 35(6), 616-628. https://doi.org/10.1016/j.csi.2013.04.002

Holliday, M. (2001). Coaching, Mentoring and Managing: A Coach Guide Book. Franklin Lakes, NJ: Career Press

ISO. (2001). Software engineering - Product quality Part 1: Quality model

ISO. (2012). Information technology - Process assessment - Part 5: An exemplar software life cycle process assessment model

Kaltio, T., Kinnula, A. (2000). Deploying the defined SW process. Software Process: Improvement and 
Practice, 5(1), 65-83. https://doi.org/10.1002/ (SICI) 1099-1670(200003)5:1<65::AIDSPIP112>3.0.CO;2-R

Kontio, J. Bragge, J., Lehtola, L. (2008). The focus group method as an empirical tool in software engineering. En F. Shull, J. Singer \& D. Sjøberg (Eds.), Guide to Advanced Empirical Software Engineering (pp. 93-116): Springer London

Marcos, E. (2002). Investigación en ingeniería del software vs. desarrollo software. MIFISIS

Massey, A. P., Montoya-Weiss, M. (1997). A knowledge exchange perspective of technology transfer. En Proceedings of the Thirtieth Hawaii International Conference on System Sciences. https://doi. org/10.1109/HICSS.1997.661576

McKay, J., Marshall, P. (2001). The dual imperatives of action research. Information Technology \& People, 14, 46-59. https://doi. org/10.1108/09593840110384771

Mendoza, M., González, C., Pino, F. J. (2013). Focus group como proceso en ingeniería de software: una experiencia desde la práctica. Dyna, 80(181), 51-60 Montoni, M., Santos, G., Rocha, A., Figueiredo, S., Cabral, R., Barcellos, R., Barreto, A., Soares, A., Cerdeiral, C., Lupo, P. (2006). Taba workstation: Supporting software process deployment based on CMMI and MR-MPS.BR. Product-Focused Software Process Improvement, 4034, 249-262. https://doi. org/10.1007/11767718_22

Nonaka, I., Toyama, R. (2003). The knowledge-creating theory revisited: Knowledge creation as a synthesizing process. Knowledge Management Research \& Practice, 1(1), 2-10. https://doi.org/10.1057/palgrave.kmrp.8500001

Oktaba, H. J., Morales, M. E., Dávila, M. (2012). KUALI-BEH: Software Project Common Concepts, versión 1.1. Universidad Nacional Autónoma de México

Patiño, N., Zambrano, F. (2014a). Método para el despliegue de procesos en el contexto de las pequeñas organizaciones desarrolladoras de software [Tesis de grado]. Universidad del Cauca, Popayan, Colombia
Patiño, N., Zambrano, F. (2014b). Método para el despliegue de procesos en el contexto de las pequeñas organizaciones desarrolladoras de software - Guía electrónica de proceso, V. 1.2. http://artemisa.unicauca.edu.co/ avogfjzj/deploy/epg\%20v1.2/

Read, C. (2008). Scaffolding children's learning through story and drama. IATEFL Young Learner Publication 2

Sanchez-Segura, M.-I., Medina-Dominguez, F., Amescua, A., Mora-Soto, A. (2010). Improving the efficiency of use of software engineering practices using product patterns. Information Sciences, 180(14), 2721-2742. https://doi.org/10.1016/j.ins.2010.03.028

SEI. (2010). CMMI for Development, Version 1.3 (CMU/ SEl-2010-TR-033). Pittsburgh, PA: Software Engineering Institute; Carnegie Mellon University

Serrano, C. (2009). Modelo integral para el profesional en ingeniería: Universidad del Cauca

Sousa, F., Aparicio, M., Costa, C. (2010). Organizational wiki as a knowledge management tool. En Proceedings of the 28th ACM International Conference on Design of Communication, 33-39. https://doi. org/10.1145/1878450.1878457

Uchihira, N. (2006). Internalization method of R\&D project management knowledge in stage gate analysis. IAMOT. http://park1.aeonnet.ne.jp/ naoshi.uchihira/IAMOT-uchihira-proc.pdf

Uchihira, N., Hirabayashi, Y., Sugihara, T., Hiraishi, K., Ikawa, Y. (2012). Knowledge transfer in R\&D project management: Application to business-academia collaboration project. En Proceedings of PICMET 12: Technology Management for Emerging Technologies

Walter, D. (2001). Training on the Job: A New Team-Driven Approach That Empowers Employees, Is Quick to Implement, Gets Bottom-Line Results. American Society for Training and Development

Wigg, K. (2004). People-Focused Knowledge Management: How Effective Decision Making Leads to Corporate Success. Routledge

Young, R. (2010). Knowledge Management Tools and Techniques Manual. Tokyo: Asian Productivity Organization 